\title{
A Phase 2 Study of Combination Therapy With Arsenic Trioxide and Gemtuzumab Ozogamicin in Patients With Myelodysplastic Syndromes or Secondary Acute Myeloid Leukemia
}

\author{
Mikkael A. Sekeres, MD, MS'; Jaroslaw P. Maciejewski, MD, PhD'; Harry P. Erba, MD²; Manuel Afable, MD; \\ Ricki Englehaupt'; Ronald Sobecks, MD'; Anjali Advani, MD'; Sherry Seel'; Josephine Chan, PhD'; \\ and Matt E. Kalaycio, $\mathrm{MD}^{1}$
}

BACKGROUND: Higher-risk myelodysplastic syndromes (MDS) are similar pathobiologically to acute myeloid leukemia (AML), particularly in older adults. AML therapies thus may have activity in MDS. In the current study, phase 2 study data of arsenic trioxide (ATO) and gemtuzumab ozogamicin (GO) in CD33-positive patients with MDS and secondary AML (SAML) were presented. METHODS: Between June 2004 and February 2006, 30 patients with higherrisk MDS or SAML received ATO (at a dose of $0.25 \mathrm{mg} / \mathrm{kg}$ intravenously for 5 days during Week 1, then twice weekly during Weeks 2-12) and GO (at a dose of $3 \mathrm{mg} / \mathrm{m}^{2}$ on Day 8) for 1 or 2 cycles of 12 weeks each. The primary endpoint was response as per MDS or AML International Working Group (IWG) criteria. Adverse events were collected throughout treatment. Patients were followed for a minimum of 3 years for survival. RESULTS: The median patient age was 69 years. A total of 18 patients had MDS, 12 had SAML, and 19 had been previously treated. Seventeen patients (57\%) completed $\geq 1$ cycle, and 7 patients (23\%) completed 2 cycles. IWG responses occurred in 9 patients (30\%) according to IWG MDS criteria (including 2 of 7 patients who failed hypomethylating agents) and 3 of 12 AML patients (25\%) according to IWG AML criteria. Grade 3/4 (according to National Cancer Institute Common Toxicity Criteria [version 3.0]) thrombocytopenia occurred in $47 \%$ of patients, neutropenia in $63 \%$, and anemia in $37 \%$ of patients. The median overall survival was 9.7 months (28.6 months in responders and 7.6 months in nonresponders; $P$ $<.001)$. Patients who completed 2 cycles of therapy spent a median of 13 days in the hospital. CONCLUSIONS: Combination therapy with ATO and GO was found to have acceptable response rates and toxicity, and may be a viable treatment option to standard induction therapy, particularly for patients who fail therapy with hypomethylating agents. Cancer 2011;117:1253-61. (c) 2010 American Cancer Society.

KEYWORDS: acute myeloid leukemia, myelodysplastic syndromes, clinical trial, arsenic trioxide, gemtuzumab ozogamicin.

The myelodysplastic syndromes (MDS) represent a spectrum of bone marrow disorders now considered to be cancers by the Surveillance, Epidemiology, and End Results (SEER) program of the National Cancer Institute, with a yearly incidence rate in the United States of 3.4 per 100,000 citizens, or $>10,000$ new diagnoses yearly. ${ }^{1}$ Within subcategories of MDS, refractory anemia (RA), refractory cytopenia with multilineage dysplasia with or without ringed sideroblasts, or MDS associated with an isolated del(5q) abnormality can persist for years, and are considered lower-risk MDS, representing the majority of MDS diagnoses. ${ }^{2-5}$ As oncogenes are activated and tumor suppressor genes inactivated, genetic lesions

Corresponding author: Mikkael A. Sekeres, MD, MS, Leukemia Program, Department of Hematologic Oncology and Blood Disorders, Cleveland Clinic Taussig Cancer Institute, Desk R35, 9500 Euclid Avenue, Cleveland, OH 44195; Fax: (216) 636-0636; sekerem@ccf.org

${ }^{1}$ Leukemia Program, Department of Hematologic Oncology and Blood Disorders, Taussig Cancer Institute, Cleveland Clinic, Cleveland, Ohio; ${ }^{2}$ University of Michigan, Ann Arbor, Michigan

Presented in part at the American Society of Hematology Annual Meeting, December 9-12, 2006, Atlanta, GA and published as Sekeres MA, Maciejewski J, Erba $\mathrm{H}$, Sobecks R, Advani A, Nichols J, Chen J. A phase II trial of combination therapy with arsenic trioxide (ATO) and gemtuzumab ozogamicin (GO) in patients with high-risk myelodysplastic syndromes (MDS) or acute myeloid leukemia (AML) arising from MDS [poster]. Blood 2006;108:754a.

DOI: 10.1002/cncr.25686, Received: April 30, 2010; Revised: July 16, 2010; Accepted: August 30, 2010, Published online October 19, 2010 in Wiley Online Library (wileyonlinelibrary.com) 
accumulate and lower-risk MDS becomes proliferative or higher-risk MDS, and eventually acute myeloid leukemia (AML), believed to be facilitated through ras, FMS, and p53 mutations. ${ }^{6}$ RA with excess blasts (RAEB) or RAEB in transformation to leukemia (RAEB-T, $)^{7}$ run accelerated courses, and are referred to as higher-risk MDS. ${ }^{7-11}$

Bone marrow findings in patients with higher-risk MDS are similar to those found in patients with AML, particularly AML occurring in older adults. MDS-associated myeloblasts are just as likely to express CD33, which is found in $90 \%$ of patients with AML. ${ }^{12}$ Moreover, adults with higher-risk MDS demonstrate striking cytogenetic similarities to older adults with AML. ${ }^{13-15}$ Thus, therapies that are effective in patients with AML may also demonstrate activity in patients with advanced MDS and secondary AML (sAML), and outpatient regimens in particular have appeal over standard remission induction therapies, which have limited efficacy and require a prolonged inpatient stay. ${ }^{16}$

Gemtuzumab ozogamicin (GO) is an anti-CD33 monoclonal antibody covalently linked to calicheamicin, a cytotoxic agent. It causes double-strand DNA breaks when internalized into the myeloblast nucleus, and results in a complete remission (CR) or CR with incomplete platelet recovery (CRi) in approximately $29 \%$ of older AML patients in first recurrence. ${ }^{17,18}$ Arsenic trioxide (ATO) works through apoptotic and pro-differentiating mechanisms, and has been shown to inhibit the production of vascular endothelial growth factor. ${ }^{19-21}$ Used as a single agent in patients with MDS, it produced a hematologic response rate of approximately $20 \% .^{22,23}$

On the basis of the data demonstrating limited efficacy using ATO and GO as single agents in patients with AML and MDS, we conducted a phase 2 study of combination therapy in patients with higher-risk MDS or AML arising from MDS (sAML) because ATO may downregulate inhibitors of apoptosis in bone marrow blasts, allowing for enhanced cytotoxicity by GO.

\section{MATERIALS AND METHODS}

\section{Patients}

This multicenter trial was approved by the institutional review boards of the Cleveland Clinic and the University of Michigan, and conducted from February 2004 through June 2006. Enrolled patients had to have a diagnosis of higher-risk MDS, defined as an International Prognostic Scoring System (IPSS) score $\geq 1.5$ or a French-AmericanBritish (FAB) or World Health Organization (WHO)

\section{GO $3 \mathrm{mg} / \mathrm{m}^{2}$ day 8}

ATO $0.25 \mathrm{mg} / \mathrm{kg} \times 5$ days and $0.25 \mathrm{mg} / \mathrm{kg}$ twice a week for 11 weeks

Total 1-2 Cycles

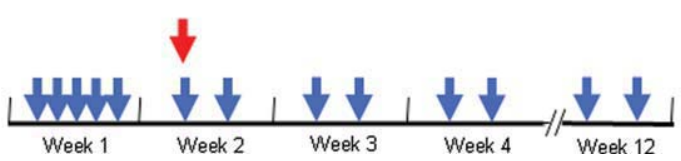

Figure 1. Treatment schema is shown. GO indicates gemtuzumab ozogamicin; ATO, arsenic trioxide. [Color figure can be viewed in the online issue, which is available at wileyonlinelibrary.com.]

pathologic classification with $\geq 5 \%$ CD33-positive myeloblasts or AML arising from MDS (with CD33-positive myeloblasts) and not have received previous remission induction therapy. ${ }^{2-4,24}$ Cytogenetic analyses were based on $\geq 20$ metaphase divisions, and risk classifications were performed according to IPSS designations for MDS patients and per Cancer and Leukemia Group B (CALGB) 8461 for AML patients. ${ }^{25}$ All diagnoses were confirmed at 1 of the 2 participating institutions. Additional inclusion criteria were age $>18$ years; an Eastern Cooperative Oncology Group status of 0, 1, or 2; serum potassium $\geq 4.0 \mathrm{mEq} / \mathrm{dL}$ and serum magnesium $>1.8$ $\mathrm{mg} / \mathrm{dL}$; and an absolute QTc interval $<460$ milliseconds. Patients could not be pregnant or lactating; could not be candidates for bone marrow transplantation or have undergone bone marrow transplantation in the past; must not have received another investigational or approved therapy for MDS within 4 weeks of study enrollment, including growth factors; must not have received prior therapy with either ATO or GO; must have had a life expectancy of at least 4 months; must have had adequate renal and hepatic function; and must not have had a prior history of malignancy other than MDS unless free of disease for $\geq 3$ years. This trial was registered with clinicaltrials.gov identifier \#NCT00274781.

\section{Study Design}

Although to our knowledge there are no phase 1 data establishing a maximum tolerated dose for the combination of ATO and GO, toxicities of the 2 agents were believed to be sufficiently non-overlapping to support empiric combination dosing based on previous studies of the single agents. Thus, ATO dosing was based on the phase 2 European Union regimen ${ }^{23}$ at a dose of $0.25 \mathrm{mg} /$ 
kg administered intravenously on Days 1 through 5 during Week 1 and then twice weekly during Weeks 2 to 12 , and GO at a dose of $3 \mathrm{mg} / \mathrm{m}^{2}$ on Day 8 for 1 or 2 cycles of 12 weeks each (Fig. 1). The doses of GO were similar to those used in phase 1 studies in patients with recurrent or refractory AML in which patients achieved a $\mathrm{CR},{ }^{18}$ yet were lower than the standard dose of $9 \mathrm{mg} / \mathrm{m}^{2}$ given to patients with MDS in a phase 2 , multicenter trial to minimize toxicity. Regular conferences were held in which the investigators reviewed toxicities and made a determination of whether study continuation was ethical. One such mandated conference occurred after enrollment of the first 6 patients to determine whether toxicities were acceptable to continue enrollment. Patients who demonstrated stable disease or improvement on a Week 12 bone marrow biopsy received a second 12 -week cycle, and then another Week 24 biopsy. Patients did not receive erythropoiesis-stimulating agents or growth factors while on the study. Toxicities were assessed using the National Cancer Institute Common Toxicity Criteria (version 3.0) over the entire course of therapy.

\section{Statistics and Efficacy Assessments}

The primary endpoint was response as defined by the Modified International Working Group (IWG) criteria for $\mathrm{MDS}^{26}$ or, for AML patients, the IWG response criteria for AML. ${ }^{27}$ The null hypothesis to be tested was that the percentage of patients who will respond to combination GO and ATO therapy is $<10 \%$. A total of $\geq 9$ responses observed in 30 evaluable patients was taken as evidence warranting further study of the regimen, provided the toxicity profile also appears favorable. This design had a significance level (probability of falsely declaring that ATO warrants further study when the true response rate is $\leq 10 \%$ ) of .10 , and power (probability of correctly declaring that the combination of GO and ATO warranted further study when the true response rate was $30 \%)$ of 0.80 . Baseline characteristics were reported as medians with ranges, as was survival, which was measured from the date of study enrollment until death or study closure for follow-up (March 2009). Survival differences were calculated using the Student $t$ test.

\section{RESULTS}

\section{Baseline Characteristics}

Thirty patients were enrolled; baseline characteristics are presented in Table 1. The median age was 69 years, and 9 patients $(30 \%)$ were female. Eighteen patients $(60 \%)$ had
MDS (5 with RAEB-1, 10 with RAEB-2, and 3 with CMML-2,). Twelve patients (40\%) had AML with cytogenetic risk groups as defined by CALGB 8461: intermediate (7 patients), adverse (3 patients), and unknown/no growth (2 patients). IPSS classifications for all patients were high (16 patients), intermediate-2 (9 patients), intermediate-1 (3 patients), and unclassifiable (2 patients), whereas IPSS cytogenetic risk classifications for MDS patients were good (6 patients), intermediate (4 patients), poor (4 patients), and no growth (4 patients). Patients had a median of $15 \%$ blasts (11.5\% for those with MDS and $43.5 \%$ for those with AML). Patients carried a diagnosis of MDS/AML a median of 5 months before trial entry. Fifteen patients received prior therapies, including growth factors (10 patients), azacitidine (5 patients), hydroxyurea (3 patients), prednisone (1 patient), anagrelide (1 patient), thalidomide (1 patient), and lenalidomide (1 patient).

\section{Treatment Tolerability}

Of the 30 patients enrolled, 17 (57\%) completed a single 12 -week cycle of therapy, 7 of whom (23\%) completed two 12 -week cycles. Reasons for discontinuing therapy during the first cycle included patient/physician choice (5 patients), progressive disease ( 5 patients), or treatment toxicity (3 patients). Reasons for discontinuing therapy after the first cycle included progressive disease (8 patients) and toxicity (2 patients). Overall, adverse events of any grade were experienced by $100 \%$ of patients (Table 2). Serious (grade 3 or 4) hematologic events occurred in $63 \%$ of patients, as would be expected with myelotoxic therapy, and included neutropenia in 19 patients (63\%), thrombocytopenia in 14 patients (47\%), and anemia in 11 patients (37\%). Serious nonhematologic toxicities included pneumonia in 5 patients (17\%), fatigue in 4 patients (13\%), dyspnea in 4 patients (13\%), QT/QTc prolongation in 3 patients (10\%), and gastrointestinal toxicities in 2 patients (7\%).

\section{Treatment Efficacy}

Responses occurred in 9 patients (30\%) (Table 3) according to the IWG MDS criteria, in 3 of 12 AML patients (25\%) according to the IWG AML criteria, and in 3 patients (10\%) by both criteria. Using the modified IWG MDS criteria, 3 patients achieved a partial response, 3 achieved a neutrophil response, 2 achieved a platelet response, and 1 achieved both platelet and neutrophil responses. Using the IWG AML criteria, 3 patients with AML achieved a partial response. Two of 7 patients previously treated with hypomethylating agents, such as 
Table 1. Baseline Characteristics

Demographic Category

Total No. of Patients

Age, $y$

Median

Range

$\operatorname{MDS}(n=18)$

Lower risk (IPSS low or intermediate-1) $(n=3)$

Median

Range

Higher risk (IPSS intermediate-2 or high) $(n=15)$

Median

Range

AML $(n=12)$

Median

Range

Sex

Male

Female

WHO category

RAEB-1

RAEB-2

CMML-2

SAML

IPSS risk category $(n=30)$

Lower risk

Low

Intermediate-1

Higher risk

Intermediate-2

High

Unclassifiable

Time from diagnosis to treatment $(n=30)$, mo

Lower risk

Median

Higher risk

Median

Baseline cytopenias

Anemia

Neutropenia

Thrombocytopenia

Previous therapies

Growth factors

Azacitidine

Hydroxyurea

Lenalidomide, thalidomide, anagrelide, and prednisone (each: $n=1$ )
No. of Patients

30

69

44-88

67

44-73

69

$54-80$

77

$58-88$

$21 \quad 70 \%$

$9 \quad 30 \%$

$5 \quad 17 \%$

$10-33 \%$

$3 \quad 10 \%$

$12 \quad 40 \%$

MDS indicates myelodysplastic syndromes; IPSS, International Prognostic Scoring System; AML, acute myeloid leukemia; WHO, World Health Organization; RAEB, refractory anemia with excess blasts; CMML, chronic myelomonocytic leukemia; sAML, secondary AML. 
Table 2. Adverse Events

\begin{tabular}{|c|c|c|c|c|}
\hline \multirow{2}{*}{$\begin{array}{l}\text { Adverse Events }(n=30) \\
\text { Event }\end{array}$} & \multicolumn{2}{|c|}{$\begin{array}{l}\text { Patients with } \\
\text { Any Event }\end{array}$} & \multicolumn{2}{|c|}{$\begin{array}{l}\text { Patients with } \\
\text { Grade 3/4 Event }\end{array}$} \\
\hline & No. & $\%$ & No. & $\%$ \\
\hline \multicolumn{5}{|l|}{ Hematologic adverse events } \\
\hline Thrombocytopenia & 16 & $53 \%$ & 14 & $47 \%$ \\
\hline Neutropenia & 20 & $67 \%$ & 19 & $63 \%$ \\
\hline Anemia & 22 & $73 \%$ & 11 & $37 \%$ \\
\hline \multicolumn{5}{|l|}{ Nonhematologic adverse events } \\
\hline Fatigue & 28 & $93 \%$ & 4 & $13 \%$ \\
\hline Rash/pruritus & 12 & $40 \%$ & 0 & $0 \%$ \\
\hline Pain & 16 & $53 \%$ & 0 & $0 \%$ \\
\hline Edema & 9 & $30 \%$ & 0 & $0 \%$ \\
\hline Nausea/vomiting/dyspepsia & 18 & $60 \%$ & 1 & $3 \%$ \\
\hline Diarrhea/constipation & 20 & $67 \%$ & 1 & $3 \%$ \\
\hline Dyspnea & 15 & $50 \%$ & 4 & $13 \%$ \\
\hline Anorexia/decreased appetite & 10 & $33 \%$ & 0 & $0 \%$ \\
\hline Pyrexia & 15 & $50 \%$ & 0 & $0 \%$ \\
\hline Cough & 11 & $37 \%$ & 0 & $0 \%$ \\
\hline Insomnia & 4 & $13 \%$ & 0 & $0 \%$ \\
\hline Catheter/infusion site disorders/infections & 4 & $13 \%$ & 0 & $0 \%$ \\
\hline Abdominal pain & 6 & $20 \%$ & 0 & $0 \%$ \\
\hline Cardiac arrhythmia & 8 & $27 \%$ & 0 & $0 \%$ \\
\hline Pneumonia & 8 & $27 \%$ & 5 & $17 \%$ \\
\hline Headache & 11 & $37 \%$ & 0 & $0 \%$ \\
\hline QT/QTc interval prolongation & 4 & $13 \%$ & 3 & $10 \%$ \\
\hline Chills/rigors & 16 & $53 \%$ & 0 & $0 \%$ \\
\hline Dizziness/vertigo & 6 & $20 \%$ & 0 & $0 \%$ \\
\hline Ecchymosis/petechiae & 5 & $17 \%$ & 0 & $0 \%$ \\
\hline Neuropathy & 2 & $7 \%$ & 0 & $0 \%$ \\
\hline
\end{tabular}

${ }^{a}$ Graded according to National Cancer Institute Common Toxicity Criteria (version 3.0).

Table 3. Responses

\begin{tabular}{|c|c|}
\hline Characteristic & $\mathbf{N}=30(\%)$ \\
\hline IWG MDS responses $(n=30)^{a}$ & $9(30)$ \\
\hline PR & $3(33)$ \\
\hline $\mathrm{HI}-\mathrm{N}$ & $3(33)$ \\
\hline HI-PIt & $2(22)$ \\
\hline HI-PIt $+\mathrm{N}$ & $1(11)$ \\
\hline SD/PD & 21 \\
\hline IWG AML responses $(n=12)^{a}$ & $3(25)$ \\
\hline PR & $3(100)$ \\
\hline SD/PD & 9 \\
\hline
\end{tabular}

IWG indicates International Working Group; MDS, myelodysplastic syndromes; PR, partial response; HI, hematologic improvement; N, neutrophils; Plt, platelets; SD, stable disease; PD, progressive disease; AML, acute myeloid leukemia.

${ }^{a}$ Three patients fulfilled criteria for a PR according to both IWG MDS and AML assessments.

azacitidine and decitabine, also achieved IWG responses. Individual patient data appear in Table 4.

The overall median survival for the entire study cohort was 9.7 months (Table 5). Responders survived a median of 28.6 months (range, 3.5 months-38 months), whereas nonresponders survived a median of 7.6 months (range, 0.75 months-38 months; $P<.001$ ). For the 2 lower-risk patients who completed more than one 12week cycle of therapy, the median survival was 11.3 months (range, 6.5 months- 16 months); for the 7 higherrisk patients who completed $\geq 1$ cycle of therapy, the median survival was 10.3 months (range, 3.5 months-38 months), whereas for those who completed 2 cycles, it was 18.8 months (range, 8 months-38 months). For the 8 patients with AML who completed $\geq 1$ cycle of therapy, the median survival was 5.1 months (range, 2.5 months23 months), whereas for those patients who completed $\geq 2$ cycles, the median survival was 21.3 months (range, 19 months-23 months). Patients who completed 2 cycles of therapy spent a median of 13 days in the hospital.

\section{DISCUSSION}

With 3 drugs approved by the US Food and Drug Administration for the treatment of MDS as single agents, the era of combination therapy has arrived, particularly for 


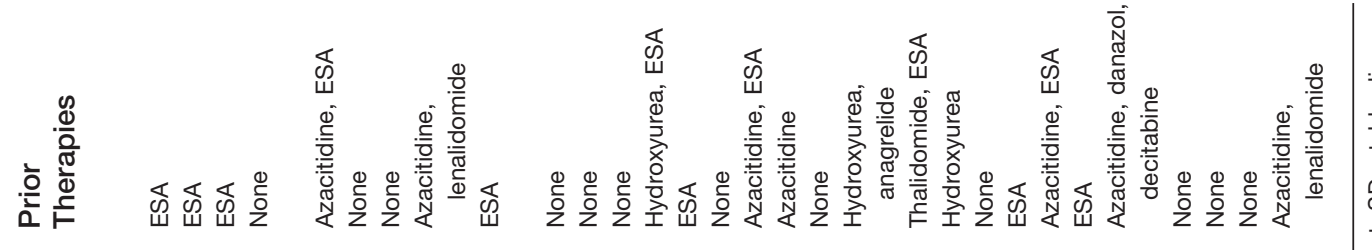
○

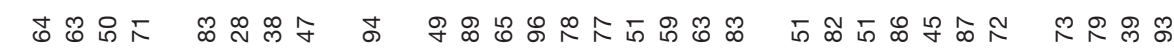

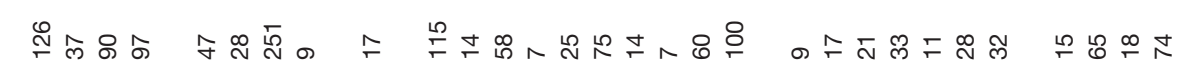

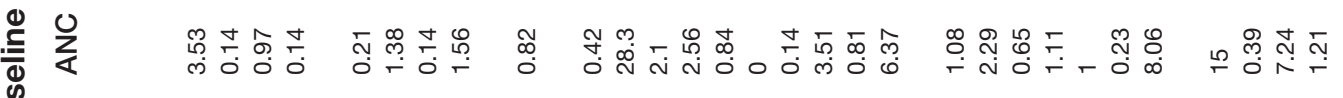

๓ి

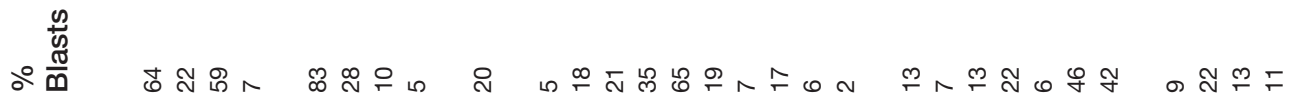

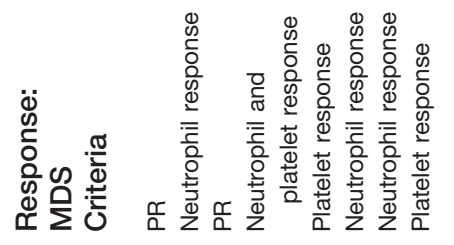

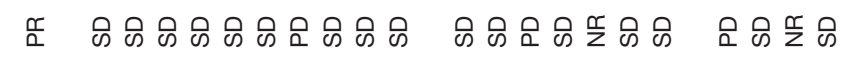

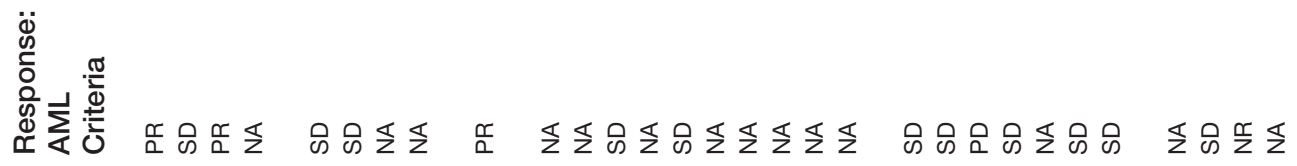

ర্

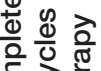

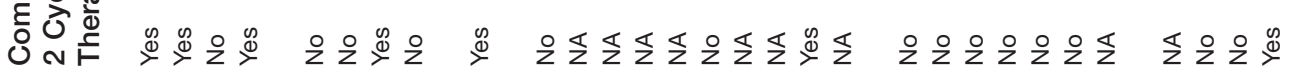

¿

응 응 웅

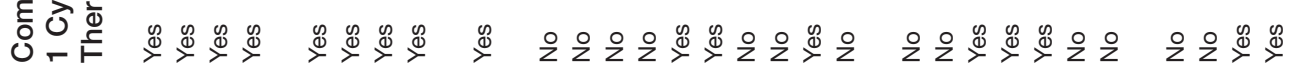

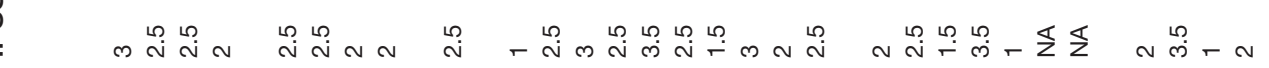

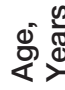

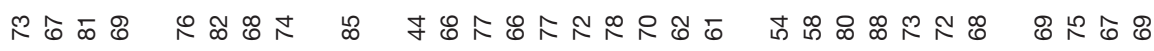

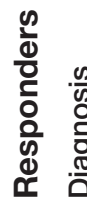

离岀

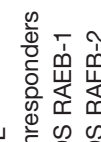

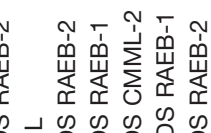

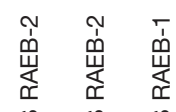

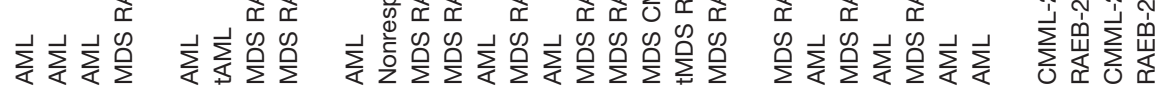


Table 5. Overall Survival

\begin{tabular}{|c|c|c|}
\hline Cohort & $\begin{array}{l}\text { No. of } \\
\text { Patients }\end{array}$ & $\begin{array}{l}\text { Median Survival } \\
\text { (Range), Months }\end{array}$ \\
\hline All patients & 30 & $9.7(0.75-38)$ \\
\hline Responders & 9 & $28.6(3.5-38)$ \\
\hline Nonresponders & 21 & $7.6(0.75-38)$ \\
\hline \multicolumn{3}{|c|}{ Patients completing $\geq 1$ cycle of therapy $(n=17$ ) } \\
\hline \multicolumn{3}{|l|}{ MDS } \\
\hline \multicolumn{3}{|l|}{ Lower risk } \\
\hline$\geq 1$ cycle & 2 & $11.3(6.5-16)$ \\
\hline$\geq 2$ cycles & & NA \\
\hline \multicolumn{3}{|l|}{ Higher risk } \\
\hline$\geq 1$ cycle & 7 & $10.3(3.5-38)$ \\
\hline$\geq 2$ cycles & 4 & $18.8(8-38)$ \\
\hline \multicolumn{3}{|l|}{ AML } \\
\hline$\geq 1$ cycle & 8 & $5.1(2.5-23)$ \\
\hline$\geq 2$ cycles & 3 & $21.3(19-23)$ \\
\hline
\end{tabular}

MDS indicates myelodysplastic syndromes; NA, not applicable; AML, acute myeloid leukemia.

those patients who have already failed 1 of these diseasemodifying therapies, and for the particularly at-risk population of patients with sAML arising out of MDS.

We studied a novel combination of drugs, each of which has demonstrated activity in MDS and/or AML. The justification for this strategy rested on the hypothesis that patients with higher-risk MDS and sAML, in which pro-proliferative mechanisms of disease potentiation predominate, and in whom excess myeloblasts (evident in every patient enrolled onto the current study) are just as likely to express CD33 as are myeloblasts in patients with AML, also retained properties of the lower-risk MDS from which their disease likely evolved, in which bone marrow microenvironment and a block in differentiation also play major roles. Thus, the use of 2 agents with complementary mechanisms of action may yield additive benefit.

The combination of ATO and GO was found to be well tolerated. It had a toxicity profile that was manageable, with the expected hematologic adverse events that accompany myelosuppressive therapy and nonhematologic toxicities occurring at an expected rate for this population of older adults with advanced disease, and similar to that noted in other studies of disease-modifying therapies in older, higher-risk populations with MDS and sAML. Because this was designed to be a phase 2 study, no dose escalation was planned; given the acceptable toxicity profile, it would be reasonable to administer GO at higher doses (such as $9 \mathrm{mg} / \mathrm{m}^{2}$ once or twice per cycle) in subsequent phase studies.
The combination of ATO and GO also was found to be effective, yielding partial and hematologic responses, particularly with regard to neutrophil and platelet counts, in $30 \%$ of patients. There were no discernable clinical characteristics that would have distinguished responders from nonresponders a priori. This response rate compared favorably with the hematologic improvement rate of 19\% (17\% in higher-risk patients) noted in a European phase 2 study by Vey et al of arsenic trioxide in MDS, and with a US study by Schiller et al reporting a hematologic improvement rate of $20 \% \quad 6 \%$ in higherrisk patients), with similar toxicities. ${ }^{22,23}$

Among those patients who had been treated previously with azacitidine, 2 of 7 experienced an IWG hematologic improvement, which is comparable to the response rate noted with, for example, clofarabine in patients similarly exposed to hypomethylating agents. ${ }^{28}$ Also similar to the azacitidine survival study, patients treated for a longer period of time appeared to have a better survival, although to some extent this may have been a self-fulfilling prophesy, because these patients also tended to be responders.

Perhaps most importantly, for patients with higherrisk MDS and sAML who might reasonably be treated with remission-inducing, cytarabine-based therapy as inpatients, this combination may be a viable outpatient alternative. In the current study, patients treated for the longest period of time spent a median of only 13 days in the hospital, compared with the initial 4 to 6 weeks they would have spent had they undergone cytoreductive therapy.

At the time this study was conducted, azacitidine had not yet been demonstrated to improve survival in a similar patient population when compared with conventional care regimens ${ }^{16}$; thus, we considered it ethical to enroll such patients onto this combination as front-line therapy. The azacitidine study established overall survival as the benchmark for efficacy in the higher-risk MDS/ AML population. The median overall survival in the current study was similar to the population treated on the azacitidine study. Whether there is an advantage of the combination regimen or single-agent azacitidine, in terms of toxicities or efficacy, can only be determined in a comparative study.

Another viable outpatient approach would be to treat older AML patients with low-dose cytarabine, as has been done by the United Kingdom Medical Research Council (MRC). ${ }^{29}$ It is difficult to make absolute direct comparisons because the majority of patients enrolled in 
the current study had MDS and not AML, and the AML patients enrolled on this study all had sAML, a group that comprised only $26 \%$ of the patients enrolled in the study by Burnett et al. Thus, the CR rate of $18 \%$ for low-dose cytarabine in patients with AML in the MRC study by Burnett et al would not necessarily translate to the population being studied herein. The median overall survival for patients enrolled in the current study who achieved a response, 28.6 months, is favorable when compared with the median overall survival of CR patients enrolled in the study by Burnett et al ( 575 days). Nevertheless, it is difficult to know whether, had we designed a phase 3 study, the combination would be any better than low-dose cytarabine. Future directions for this combination include a comparison with standard, cytarabine-based remission induction therapy in patients with sAML, and with other drugs being explored in patients with higher-risk MDS who have developed disease recurrence or are refractory to hypomethylator therapy. Each of these drugs individually is being explored in combination with azacitidine in ongoing clinical trials; it would be equally reasonable to explore the combination with a hypomethylating agent.

In conclusion, the combination of GO and ATO is a viable alternative to other outpatient regimens for the treatment of patients with higher-risk MDS and sAML. Responses were similar in de novo and previously treated patients, and a natural next step would be to develop this regimen in patients who have failed or progressed through hypomethylation or other disease-modifying therapy.

\section{CONFLICT OF INTEREST DISCLOSURES}

Supported in part by National Institutes of Health grant U54RR19397-03 (to M.A.S. and J.P.M.)

\section{REFERENCES}

1. Ma X, Does M, Raza A, Mayne ST. Myelodysplastic syndromes: incidence and survival in the United States. Cancer. 2007;109:1536-1542.

2. Bennett JM, Catovsky D, Daniel MT, et al. Proposals for the classification of the myelodysplastic syndromes. $\mathrm{Br} J$ Haematol. 1982;51:189-199.

3. Greenberg P, Cox C, LeBeau MM, et al. International scoring system for evaluating prognosis in myelodysplastic syndromes. Blood. 1997;89:2079-2088.

4. Vardiman J, Thiele J, Arber D, et al. The 2008 revision of the World Health Organization (WHO) classification of myeloid neoplasms and acute leukemia: rationale and important changes. Blood. 2009;114:937-951.

5. Sekeres MA, Schoonen WM, Kantarjian H, et al. Characteristics of US patients with myelodysplastic syndromes: results of six cross-sectional physician surveys. J Natl Cancer Inst. 2008; 100:1542-1551.
6. Sugimoto K, Hirano N, Toyoshima H, et al. Mutations of the p53 gene in myelodysplastic syndrome (MDS) and MDS-derived leukemia. Blood. 1993;81:3022-3026.

7. Harris NL, Jaffe ES, Diebold J, et al. The World Health Organization classification of neoplastic diseases of the hematopoietic and lymphoid tissues. Report of the Clinical Advisory Committee meeting, Airlie House, Virginia, November, 1997. Ann Oncol. 1999;10:1419-1432.

8. Vallespi T, Torrabadella M, Julia A, et al. Myelodysplastic syndromes: a study of 101 cases according to the FAB classification. Br J Haematol. 1985;61:83-92.

9. Foucar K, Langdon RM, Armitage JO, Olson DB, Carroll TJ. Myelodysplastic syndromes. A clinical and pathologic analysis of 109 cases. Cancer. 1985;56:553-561.

10. Economopoulos T, Stathakis N, Foudoulakis A, et al. Myelodysplastic syndromes: analysis of 131 cases according to the FAB classification. Eur J Haematol. 1987;38:338-344.

11. Kerkhofs H, Hermans J, Haak HL, Leeksma CH. Utility of the FAB classification for myelodysplastic syndromes: investigation of prognostic factors in 237 cases. Br J Haematol. 1987;65:73-81.

12. Appelbaum FR. Antibody-targeted therapy for myeloid leukemia. Semin Hematol. 1999;36(4 suppl 6):2-8.

13. Leith CP,Kopecky KJ, Godwin J, et al. Acute myeloid leukemia in the elderly: assessment of multidrug resistance (MDR1) and cytogenetics distinguishes biologic subgroups with remarkably distinct responses to standard chemotherapy. $A$ Southwest Oncology Group study. Blood. 1997;89:3323-3329.

14. Rossi G, Pelizzari AM, Bellotti D, Tonelli M, Barlati S. Cytogenetic analogy between myelodysplastic syndrome and acute myeloid leukemia of elderly patients. Leukemia. 2000;14:636-641.

15. Haase D, Germing U, Schanz J, et al. New insights into the prognostic impact of the karyotype in MDS and correlation with subtypes: evidence from a core dataset of 2124 patients. Blood. 2007;110:4385-4395.

16. Fenaux P, Mufti GJ, Hellstrom-Lindberg E, et al. Efficacy of azacitidine compared with that of conventional care regimens in the treatment of higher-risk myelodysplastic syndromes: a randomised, open-label, phase III study. Lancet Oncol. 2009;10:223-232.

17. Sievers EL, Larson RA, Stadtmauer EA, et al. Efficacy and safety of gemtuzumab ozogamicin in patients with CD33positive acute myeloid leukemia in first relapse. $J$ Clin Oncol. 2001;19:3244-3254.

18. Sievers EL, Appelbaum FR, Spielberger RT, et al. Selective ablation of acute myeloid leukemia using antibody-targeted chemotherapy: a phase I study of an anti-CD33 calicheamicin immunoconjugate. Blood. 1999;93:3678-3684.

19. Chen GQ, Shi XG, Tang W, et al. Use of arsenic trioxide (As2O3) in the treatment of acute promyelocytic leukemia (APL): I. As2 33 exerts dose-dependent dual effects on APL cells. Blood. 1997;89:3345-3353.

20. Chen GQ, Zhu J, Shi XG, et al. In vitro studies on cellular and molecular mechanisms of arsenic trioxide (As2O3) in the treatment of acute promyelocytic leukemia: As2O3 induces NB4 cell apoptosis with downregulation of $\mathrm{Bcl}-2$ expression and modulation of PML-RAR alpha/PML proteins. Blood. 1996;88:1052-1061.

21. Roboz GJ, Dias S, Lam G, et al. Arsenic trioxide induces dose- and time-dependent apoptosis of endothelium and may exert an antileukemic effect via inhibition of angiogenesis. Blood. 2000;96:1525-1530. 
22. Vey N, Bosly A, Guerci A, et al. Arsenic trioxide in patients with myelodysplastic syndromes: a phase II multicenter study. J Clin Oncol. 2006;24:2465-2471.

23. Schiller GJ, Slack J, Hainsworth JD, et al. Phase II multicenter study of arsenic trioxide in patients with myelodysplastic syndromes. J Clin Oncol. 2006;24:2456-2464.

24. Vardiman JW, Harris NL, Brunning RD. The World Health Organization (WHO) classification of the myeloid neoplasms. Blood. 2002;100:2292-2302.

25. Byrd JC, Mrozek K, Dodge RK, et al. Pretreatment cytogenetic abnormalities are predictive of induction success, cumulative incidence of relapse, and overall survival in adult patients with de novo acute myeloid leukemia: results from Cancer and Leukemia Group B (CALGB 8461). Blood. 2002;100:4325-4336.

26. Cheson BD, Greenberg PL, Bennett JM, et al. Clinical application and proposal for modification of the Interna- tional Working Group (IWG) response criteria in myelodysplasia. Blood. 2006;108:419-425.

27. Cheson BD, Bennett JM, Kopecky KJ, et al. Revised recommendations of the International Working Group for Diagnosis, Standardization of Response Criteria, Treatment Outcomes, and Reporting Standards for Therapeutic Trials in Acute Myeloid Leukemia. J Clin Oncol. 2003;21:4642-4649.

28. Faderl S, Garcia-Manero G, Ravandi F, et al. Oral (po) and intravenous (iv) clofarabine for patients (pts) with myelodysplastic syndrome (MDS). ASH Annual Meeting Abstracts. 2008;112:222-. Available at: http://abstracts.hematologylibrary.org/cgi/content/abstract/ashmtg;112/11/222.

29. Burnett AK Milligan D, Prentice AG, et al. A comparison of low-dose cytarabine and hydroxyurea with or without alltrans retinoic acid for acute myeloid leukemia and high-risk myelodysplastic syndrome in patients not considered fit for intensive treatment. Cancer. 2007;109:1114-1124. 